\title{
ZONAS DE LIBERDADE LGBTI+? PRÁTICAS E GRAMÁTICAS PARA UMA INTERVENÇÃO PROFISSIONAL INCLUSIVA COM CRIANÇAS E JOVENS LGBTI ${ }^{1}$ \\ Mafalda Esteres \\ Ana Cristina Santos \\ Alexandra Santos***
}

\section{Resumo}

A partir de resultados do projeto europeu «Diversidade e Infância», cuja metodologia qualitativa e quantitativa envolveu crianças, jovens e profissionais de áreas como a educação, saúde e intervenção familiar, neste artigo são identificadas boas práticas enquanto medidas de ação afirmativa no mainstreaming LGBTI+, mas também os vazios e as resistências que afetam o quotidiano de crianças e jovens LGBTI+. Conhecer as perspetivas das diferentes partes intervenientes é um passo fundamental para ajustar a implementação, monitorização e aplicação de leis e medidas que visam garantir a igualdade e a não-discriminação em função da orientação sexual, identidade e expressão de género e características sexuais.

Palavras-chave: Lei da autodeterminação de género, diversidade sexual e de género, infância, políticas públicas, Portugal.

\footnotetext{
Abstract

LGBTI+ Freedom Zones? Practices and grammars for an inclusive professional intervention with LGBTI+ children and young people

Drawing on findings from the European research project «Diversity and Childhood», whose qualitative and quantitative methodology involved children, young people and

1 Este artigo resulta de investigação financiada pela Comissão Europeia no âmbito do programa Cidadãos, Igualdade, Direitos e Valores / Projeto de investigação europeu «Diversity and Childhood: Changing social attitudes towards gender diversity in children across Europe» [856680]. Em Portugal, o projeto teve como instituições parceiras a Comissão para a Cidadania e Igualdade de Género (CIG), o Ministério da Educação e a associação rede ex aequo.

* Centro de Estudos Sociais, Universidade de Coimbra (CES-UC), Coimbra, Portugal.

Endereço postal: CES-UC, Colégio de S. Jerónimo, 3000-995 Coimbra, Portugal.

Endereço eletrónico: mafaldaesteves@ces.uc.pt

** Centro de Estudos Sociais, Universidade de Coimbra (CES-UC), Coimbra, Portugal.

Endereço postal: CES-UC, Colégio de S. Jerónimo, 3000-995 Coimbra, Portugal.

Endereço eletrónico: cristina@ces.uc.pt

*** Centro de Estudos Sociais, Universidade de Coimbra (CES-UC), Coimbra, Portugal.

Endereço postal: CES-UC, Colégio de S. Jerónimo, 3000-995 Coimbra, Portugal.

Endereço eletrónico: alexandrasantos@ces.uc.pt
} 
professionals from areas such as education, health and family intervention, in this paper the authors identify good practices as affirmative action measures in LGBTI+ mainstreaming, but also gaps and resistances that affect the daily lives of LGBTI+ children and young people. Understanding the perspectives of the different actors involved is a fundamental step to adjust the implementation, monitoring and application of laws and measures that aim to guarantee equality and non-discrimination based on sexual orientation, gender identity and expression and sexual characteristics.

Keywords: Gender self-determination law, sexual and gender diversity, childhood, public policies, Portugal.

\section{Resumen}

¿Zonas de Libertad LGBTI+? Prácticas y gramáticas para una intervención profesional inclusiva en la infancia y adolescencia LGBTI+

A partir de los resultados del proyecto europeo «Diversidad e Infancia», basado en una metodología cualitativa y cuantitativa que involucró a niños/as, jóvenes y profesionales de áreas como la educación, la salud y la intervención familiar, se identifican buenas prácticas como medidas de acción afirmativa hacia la transversalización LGBTI+, pero también brechas y resistencias que afectan la vida cotidiana en la infancia y adolescencia LGBTI+. Conocer las perspectivas de las distinctas partes involucradas es un paso fundamental para adecuar la implementación, seguimiento y aplicación de leyes y medidas que tienen como objetivo garantizar la igualdad y no discriminación por orientación sexual, identidad y expresión de género y características sexuales.

Palabras clave: Ley por la autodeterminación de género, diversidad sexual y de género, infancia, políticas públicas, Portugal.

\section{Introdução}

Em março de 2021, o Parlamento Europeu aprovou uma moção declarando a União Europeia um espaço de liberdade para pessoas lésbicas, gay, bissexuais, trans e intersexo (LGBTI+). A moção foi acompanhada da hashtag \#LGBTIQ freedomZone. A designação «espaço de liberdade» constitui uma importante medida de ação afirmativa à escala europeia em resposta ao que vinha sucedendo na Polónia desde 2019, quando algumas cidades se auto-declararam zonas livres de pessoas LGBTIQ². Este ato simbólico por parte do Parlamento Europeu dialoga com a Estratégia da União Europeia a favor de pessoas LGBTQI decidida em 2020 (UE 2020) ${ }^{3}$.

2 Para uma problematização das duas aceções do conceito de liberdade - nomeadamente uma conceção positiva de liberdade para fazer ou ser e uma conceção negativa de liberdade face a constrangimentos, ver Fahs 2014.

3 «Uma União de Igualdade: Estratégia em Favor da Igualdade das Pessoas LGBTIQ 2020-2025». Disponível em https:/ / ec.europa.eu/info/sites/default/files/lgbtiq_factsheet_2020-2025_pt.pdf [Consultado em 2 de agosto de 2021]. 
Também no contexto português é notório o compromisso público contra a discriminação. Este compromisso foi sendo consolidado durante o século XXI, com ênfase para 2018, quando foram dados passos como a aprovação da Estratégia Nacional para a Igualdade e a Não Discriminação 2018-2030 Portugal + Igual. Foi também em 2018 que uma nova lei reconheceu o direito à autodeterminação da identidade e expressão de género e à proteção das características sexuais de cada pessoa, permitindo a mudança de nome próprio e sexo legal a partir dos 16 anos e convertendo Portugal num dos poucos países alinhados com as recomendações das Nações Unidas no que respeita à proteção das características sexuais de bebés e crianças intersexo.

Tendo em conta a Estratégia europeia, bem como o compromisso do Estado português com a igualdade e anti-discriminação LGBTI+, importa agora conhecer as condições objetivas para operacionalizar este desígnio no quotidiano e, mais especificamente, nas diversas áreas de intervenção. Este artigo insere-se nesse esforço de observação e análise, visando informar mais e melhores políticas públicas e medidas de ação afirmativa direcionadas a crianças e jovens LGBTI+.

Começamos por mapear os avanços mais significativos na área das políticas públicas e legislação sobre anti-discriminação LGBTI+ no espaço português, oferecendo uma análise crítica acerca do potencial destas medidas. Seguidamente, após uma precisão sobre as metodologias adotadas, algumas das quais envolvendo crianças, apresentam-se resultados decorrentes do projeto europeu «Diversidade e Infância». Por fim, e à luz desses resultados, oferece-se uma discussão acerca do conceito de adultismo enquanto perspetiva que condiciona a implementação de medidas afirmativas na área da diversidade LGBTI+ na infância. A discussão deste conceito na reta final do artigo visa tão-só abrir portas para investigação futura nesta área, que, desejamos, venha a fortalecer o envolvimento de crianças e adolescentes em todas as fases de desenho, implementação e análise da pesquisa que lhes diz diretamente respeito. Por outras palavras, manter uma perspetiva crítica face ao adultismo foi uma das aprendizagens mais significativas que, enquanto investigadoras, fizemos a partir deste projeto.

\section{Políticas públicas e legislação sobre diversidade sexual e de género em Portugal}

Apesar do legado deixado pela ditadura mais longa da Europa ocidental, uma conjuntura política favorável associada a processos mais amplos de democratização e modernização permitiu, em Portugal, o desenvolvimento de uma ação coletiva LGBTI+ capaz de articular com decisores políticos e especialistas, fomentando a produção de conhecimento com base em evidência e, concomitantemente, informando medidas políticas de relevo (Almeida 2010; Santos 2013, 2018a, 2018b). Também a influência de instituições e organismos políticos europeus, sobretudo 
após a entrada de Portugal na União Europeia, tem contribuído para o progresso jurídico em matéria de igualdade LGBTI+.

Entre 2010-2020, registamos três mudanças legislativas significativas envolvendo conjugalidade, parentalidade e autodeterminação. Em 2010, a lei do casamento foi aprovada, seguindo-se um progressivo reconhecimento jurídico da homoparentalidade em 2016 (Santos, 2018a; 2018b), incluindo a adoção, coadoção, reprodução medicamente assistida e uma versão restritiva de gestação de substituição. Já em 2018, registam-se importantes mudanças em matéria de identidade e expressão de género, bem como em questões intersexo (Saleiro 2017; Hines e Santos 2018). Partindo da revisão da Lei de Identidade de Género de 2011, em 2018 a legislação estabeleceu a despatologização de pessoas trans, dispensando um relatório médico para pessoas com mais de 18 anos e adolescentes com mais de 16 anos de forma a mudar nome e sexo nos seus documentos. Esta lei também proibiu cirurgias injustificadas em bebés intersexo e estabeleceu que as escolas devem usar o nome social escolhido pela criança ou jovem trans ${ }^{4}$. Esta lei representa um reconhecimento por parte do Estado português de que a diversidade de género se manifesta ao longo da vida, pelo que é crucial reconhecer a expressão de género na infância e assegurar o respeito pela autodeterminação de crianças e jovens trans, intersexo e não binários (Diversity and Childhood 2020).

Data também de 2018 a Estratégia Nacional para a Igualdade e a Não Discriminação - Portugal+Igual, que inclui o Plano de ação para o combate à discriminação em razão da orientação sexual, identidade e expressão de género, e características sexuais (2018-2021) e que vem afirmar o compromisso do Estado português com a anti-discriminação ${ }^{5}$.

Relativamente ao contexto educativo em Portugal, pese embora o pendor cis-heteronormativo que informa historicamente a educação sexual em meio escolar (Saavedra et al. 2010; Duarte Silva et al. 2014; Matos et al. 2014), têm sido criados instrumentos e políticas públicas visando garantir proteção não somente das pessoas adultas, mas também das crianças e jovens LGBTI+. A criação do Estatuto do Aluno e Ética Escolar (Lei n. ${ }^{\circ}$ 51/2012 de 5 de Setembro) ${ }^{6}$ possibilita, a partir de 2012, a reivindicação do direito ao tratamento com respeito e correção por parte de qualquer membro da comunidade educativa, não podendo acontecer, em caso algum, discriminações em razão de sexo, orientação sexual e identidade de género.

4 Mais informações sobre a Lei da Identidade de Género disponíveis em https://dre.pt/pesquisa/- / search/115933863/ details/maximized

Plano de ação para o combate à discriminação em razão da orientação sexual, identidade e expressão de género, e características sexuais (2018-2021) em Estratégia Nacional para a Igualdade - Portugal + Igual (2018-2030) disponível em https://www.cig.gov.pt/wp-content/ uploads/2020/12/Resol_Cons_-Ministros_61_2018.pdf [Consultado em 5 de abril de 2021].

6 Disponível em https://dre.pt/pesquisa/-/ search/174840/details/maximized [Consultado em 1 de abril de 2021]. 
Em 2019, foi aprovado o Despacho n. ${ }^{\circ} 7247 / 2019^{7}$, que estabelece medidas para a implementação da Lei n. ${ }^{\circ}$ 38/2018 em contexto escolar e que prevê o respeito pelo nome social da/o aluna/o e o direito ao uso de uniforme e casa de banho de acordo com a sua identidade de género autodeterminada. Em 2020, foi lançada a Estratégia Nacional para os Direitos da Criança (2021-2024) ${ }^{8}$, que inclui uma preocupação em garantir bem-estar, proteção, igualdade de oportunidades e acesso aos direitos fundamentais de todas as crianças e jovens, independentemente da sua orientação sexual, identidade e expressão de género e caraterísticas sexuais.

Nos contextos de prestação de cuidados de saúde, destacamos alterações na concetualização dos processos de desenvolvimento de género, incluindo a diversidade de género na infância em Portugal, parcialmente explicados por um movimento internacional de despatologização trans forte (WPATH 2010; Suess 2020). Trata-se, portanto, de alterações que colocam o bem-estar das crianças e jovens LGBTI+ no centro das preocupações. Em 2017, a Ordem dos Psicólogos Portugueses lança o primeiro Guia Orientador da Intervenção Psicológica com Pessoas Lésbicas, Gays, Bissexuais e Trans (LGBT). Posteriormente, em 2019, o Ministério da Saúde/ Direção-Geral da Saúde lança a Estratégia de saúde para as pessoas lésbicas, gays, bissexuais, trans e intersexo: Volume 1 - Promoção da saúde das pessoas trans e inter$\operatorname{sexo}^{9}$. A criação deste importante documento contou com o envolvimento das Administrações Regionais de Saúde, Centro Hospitalar da Universidade de Coimbra - URGUS, da Comissão para a Cidadania e a Igualdade de Género - CIG, da Ordem dos Médicos, da Ordem dos Psicólogos Portugueses, da Ordem dos Enfermeiros, tendo ainda sido auscultadas associações que intervêm junto de crianças e jovens LGBTI+.

Assim, a partir do ciclo governativo iniciado em 2015, parece existir por parte do Estado um progressivo reconhecimento da relação entre a classificação da diversidade de género enquanto psicopatologia e dinâmicas de discriminação, estigmatização e violência transfóbica (Schneider 2013). Regista-se também uma mudança nas orientações da tutela visando introduzir práticas afirmativas para trabalhar com pacientes (crianças e jovens) LGBTI+, de modo a fomentar serviços de saúde inclusivos. A atual abordagem de despatologização trans informa práticas afirmativas que apoiam crianças e jovens, em consonância com estudos desenvolvidos noutros contextos (HRC 1996; Bryant 2006; Ehrensaft 2012; Green e Friedman 2013; Pyne 2014).

Disponível em https://dre.pt/home/-/dre/123962165/details/maximized [Consultado em 1 de abril de 2021].

Comissão Nacional de Promoção dos Direitos e Proteção das Crianças e Jovens. 2020. Estratégia Nacional para os Direitos da Criança (2021-2024). Disponível em https://dre.pt/application/ conteudo/151557423 [Consultado em 5 de abril de 2021].

Disponível em https://www.dgs.pt/documentos-e-publicacoes/estrategia-de-saudepara-as-pessoas-lesbicas-gays-bissexuais-trans-e-intersexo-lgbti-pdf.aspx [Consultado em 5 de abril de 2021]. 
A já mencionada Estratégia Nacional pelos Direitos da Criança (ENDC 2020-2024) reconhece a importância da proteção e promoção do bem-estar de todas as crianças, o que por sua vez se traduz num maior apoio às famílias destas crianças. Exemplos incluem o Guia para Familias de crianças com papéis e comportamentos de género diverso, produzido pela AMPLOS e Instituto de Apoio à Criança (2019), e o projeto «E se fosse outra cor» ${ }^{10}$.

Apesar destes passos significativos, fruto de transformações jurídicas e socioculturais em torno do género e da sexualidade, continuam a ser encorajadas biografias íntimas normativas (Roseneil et al. 2020). A presença de uma normatividade reprodutiva (entre outras) ligada ao género e às sexualidades explica a dificuldade em mudar o contexto sociocultural, demonstrando a insuficiência de uma intervenção estritamente jurídica. A literatura sobre regimes de bem-estar e género descreve os países da Europa do Sul como familistas, repronormativos e (hetero)normativos (Torres, Mendes e Lapa 2008; Santos 2013; Mínguez e Crespi 2017). Daí que a violência e a discriminação baseada na orientação sexual, identidade e expressão de género continuem a ser reportadas (ILGA Portugal 2019; OECD 2019; rede exaequo 2019; 2020; ILGA Europe 2021) e estejamos a assistir a retrocessos em direitos sexuais e reprodutivos adquiridos, alimentados por uma onda crescente de populismo anti-género (Möser et al. 2022).

A falta de conhecimento e formação sobre temas LGBTI+ em profissionais que trabalham na área da infância e juventude reflete estas consequências da cis-heteronormatividade, acabando por impedir uma prestação de serviços inclusiva para todas as crianças. É urgente colmatar esta lacuna, já que a violência e a discriminação baseada na LGBTIfobia comporta consequências de enorme gravidade (OECD 2019; rede ex aequo, 2019; 2020).

\section{Metodologia e discussão de resultados}

\subsection{Estratégia metodológica}

Existindo em Portugal legislação e criação de instrumentos de políticas públicas que vão ao encontro do respeito e proteção da diversidade sexual e de género na infância, quisemos conhecer as perspetivas de profissionais que atuam direta ou indiretamente junto de crianças e jovens e quais os desafios e obstáculos identificados (tanto por profissionais como por crianças), no âmbito de atuação profissional da escola, intervenção familiar e saúde, que dificultam o cumprimento pleno do quadro legal nas diferentes instituições. Pretendemos assim aprofundar o modo como a estratégia de mainstreaming LGBTI+ está a ser implementada no contexto português, identificando práticas profissionais que respondam adequadamente às crianças e jovens LGBTI+.

10 Disponível em http:/ / esefosseoutracor.com / [Consultado em 5 de Abril de 2021]. 
Para tal recolhemos informação no âmbito do projeto de investigação europeu «Diversidade e Infância» (2019-2021). Tratando-se de um projeto de investigação-ação (Chevalier e Bucles 2013), seguimos uma abordagem qualitativa recorrendo à triangulação metodológica (Flick 2005) mediante diferentes métodos de recolha, análise e interpretação da informação, de modo a colmatar as limitações decorrentes de métodos únicos (Maxwell 1996). Socorremo-nos de três fontes principais: especialistas em cada uma das áreas de intervenção do projeto (educação, saúde, media, intervenção familiar e intervenção comunitária); profissionais que trabalham de forma direta ou indireta com crianças e jovens. A cada um destes grupos foi aplicado um método distinto. Assim, realizámos 10 entrevistas qualitativas semiestruturadas a especialistas com duração média de 90 minutos; aplicámos um inquérito por questionário a profissionais $\mathrm{s}^{11}$; e realizámos uma oficina com adolescentes para discutir os temas em apreço a partir da perspetiva das crianças. Os aspetos éticos foram acautelados em todas as fases.

Através de análise temática (Braun e Clarke 2006), analisámos dimensões como perspetivas individuais sobre diversidade veiculadas por profissionais e crianças, a in/existência de políticas e medidas afirmativas aplicadas pelas instituições, e práticas profissionais adotadas. Esta análise visou identificar eventuais contradições entre a Lei da Autodeterminação de Género aprovada e a sua implementação, contribuindo assim para um diagnóstico facilitador do melhor cumprimento de medidas nesta área. Segue-se uma análise dos dados recolhidos com profissionais, a partir quer do Inquérito Europeu Diversidade e Infância, quer das entrevistas qualitativas realizadas a 10 profissionais.

2.2. Perspetivas e práticas profissionais relativamente à diversidade sexual e de género na infância - discussão de resultados

Da nossa análise resulta visível uma tendência para que profissionais que trabalham com crianças e jovens nas áreas da educação, intervenção familiar e saúde considerem o género enquanto construção social e não um conjunto de caraterísticas definidas por critérios exclusivamente biomédicos. Esta tendência encontra-se alinhada com a Lei da Autodeterminação de Género.

No entanto, foram também identificados discursos que resistem a esta mesma ideia, fazendo assentar uma definição de género em pilares biomédicos, o que revela um desencontro entre o marco jurídico vigente e a atualização de conhecimentos nesta matéria.

As respostas integram o $1^{\circ}$ Inquérito Europeu Diversidade e Infância, construído pela equipa portuguesa e validado pelo Conselho Consultivo internacional do projeto. Mais informações disponíveis em https:/ / www.diversityandchildhood.eu/project-reports [Consultado em 2 de abril de 2021]. 
É igualmente notória alguma dificuldade na distinção entre género e sexo, conceitos que são frequentemente tratados como sinónimos ou equivalentes. A esta justaposição está subjacente uma perspetiva de género binária que estrutura o género - e o sexo - de forma dicotómica. Consideramos que a conceção dicotómica de género ainda vigente introduz obstáculos face à estratégia do Estado português para a igualdade e anti-discriminação.

Nas áreas estudadas, a análise revela uma progressiva atenção dada a temas respeitantes à infância e juventude LGBTI+, verificando-se práticas profissionais afirmativas, tal como expresso na Estratégia Nacional Portugal + Igual. Esse crescente apoio demonstrado por profissionais põe em destaque a importância de criar respostas que tenham em conta as especificidades e necessidades das crianças e jovens LGBTI+, invisibilizadas e desprotegidas até 2018. Atentemos na seguinte citação:

Cada vez mais porque, primeiro porque preocupo-me com o bem-estar individual de cada um e acho muito importante e eu falo muito com eles desde o início sobre isso. Ninguém aprende num ambiente em que não está feliz, não está seguro, não se sente bem, não é confortável. Portanto, para isso acontecer tem que existir condições saudáveis entre eles, não é (...) quanto mais espaços de diálogo também existirem, nós sabemos que nem todos os ambientes são seguros. Tu podes criar isso aqui. (professora EB1, 40-44 anos)

Esta citação sugere que, se, por um lado, há uma consciencialização gradual das/os profissionais nas suas áreas de atuação, por outro, é urgente uma mudança no paradigma, em particular na forma como são pensadas ações ou estratégias dentro das instituições educativas ou nos serviços de prestação de cuidados de saúde. Uma mudança ao nível da mentalidade acompanha as anteriores mudanças. Note-se que a falta de informação sobre estas temáticas continua a fazer-se sentir, evidenciando a urgência de sensibilizar instituições e o conjunto alargado das/os profissionais para viabilizar práticas afirmativas que favorecem ambientes escolares inclusivos. Por exemplo, deve ser considerado um trabalho continuado junto do corpo docente e extensível ao corpo não docente:

[...] ainda há muitas pessoas, muitos profissionais na área da psicologia, com cabeças ainda, enfim... com atitudes pouco corretas deste ponto de vista. (Psicóloga, 55-59 anos)

[...] horrorosos, comentários do mais fascista, do mais agressivo possível, e vindo de professores, que é uma coisa que tu não devias aceitar. Vinte e dois anos a dar aulas e ainda continuamos a ouvir coisas. Vinte e dois anos e ainda continuas a ver nos livros escolares barbaridades e, por exemplo, esta questão do género tão mal defendida. (Professora EB1, 40-44 anos) 
A falta de informação sobre a temática da diversidade sexual e de género é identificada pela quase totalidade das/os participantes, dando conta da incerteza que estas/es profissionais sentem quando se pergunta se as normas de género influenciam o seu trabalho com crianças LGBTI+. Sendo verdade que estamos perante profissionais comprometidos/as em desenvolver um trabalho adequado junto das crianças e jovens LGBTI+, também é notório o acentuado desconhecimento e ausência de reflexão sobre o modo como as normas de género influenciam a forma como nos relacionamos com as crianças e os espaços onde a atividade profissional é desenvolvida.

Além disso, as/os profissionais consideraram que o medo da rejeição por parte de pares, famílias e outras pessoas adultas de referência, bem como o receio de poderem vir a ser vítimas de bullying, são alguns dos desafios que crianças e jovens LGBTI+ sentem no quotidiano, o que sublinha o caráter urgente de fazer cumprir uma estratégia de mainstreaming LGBTI+.

$\mathrm{Na}$ sua maioria, as/os profissionais reconhecem que existem situações de bullying por LGBTIfobia. Urge reforçar a corresponsabilização de profissionais visando alianças com crianças LGBTI+ nos diferentes espaços onde participam, tornando-os espaços seguros:

Nem sempre as pessoas sentem que é um espaço seguro, que toda a gente sente que é respeitada. [...] [A instituição] tem de mostrar ativamente que tem uma atitude de respeito e de inclusão, porque se não mostrar está implícito e o implícito é uma coisa pouco clara. [...] Sentirem que estão seguras para falar e para existirem, sentirem que aquele espaço é um espaço seguro para mostrarem. [...] O adulto que está lá não se pode esquecer que é um adulto e tem de ser o primeiro a passar uma mensagem de clara igualdade de circunstâncias de tratamento, de vida para todos. (Psicóloga, 55-59 anos).

Verificamos a existência de consenso entre profissionais sobre a importância da Lei da Autodeterminação de Género, nomeadamente ao nível do reconhecimento e proteção. A perceção dos profissionais é de que o Estado tem dado sinais de preocupação com o bem-estar destas crianças, o que é essencial para a redução do preconceito e violência. Consideram, contudo, que a legislação é insuficiente, carecendo de medidas de implementação no terreno.

Introduzir a temática do género e da diversidade sexual nas escolas e intervenção junto das famílias seria uma possível medida para garantir que a autodeterminação de género é cumprida nas áreas referidas. As/os profissionais sugerem ainda a realização de ações de formação ao nível curricular e profissional, maior articulação com famílias de crianças LGBTI+ e mais representação desta diversidade nos materiais (boletins de saúde infantis, campanhas de informação e sensibilização ou manuais escolares, por exemplo). Há também por parte destas/es profissionais a perceção de que as instituições nas várias áreas devem ter um papel 
proactivo e inequívoco no apoio a crianças LGBTI+, assumindo assim o dever de cumprimento do quadro legal vigente.

Tanto profissionais como crianças envolvidas no estudo consideram que, apesar da recetividade «formal» das instituições em desenvolver atividades visando temas LGBTI+, essa recetividade traduz-se em ações ou medidas insuficientes. A ausência de políticas específicas nas instituições ou de promoção de boas práticas LGBTI+ não permite a criação de espaços seguros de criatividade e autodeterminação que valorizem as opiniões, emoções e trajetórias de vida destas crianças e jovens.

É também apontada a ausência de implementação de forma sistemática e transversal, ficando as crianças e jovens LGBTI+ reféns da sensibilidade individual das/os profissionais. Este aspeto denuncia um desalinhamento sério face à estratégia de mainstreaming LGBTI+ que o Estado promove, devendo ser objeto de atenção.

Está muito dependente da pessoa e não do profissional, ou seja, se eu enquanto pessoa [es]tiver já de alguma forma desperta para estas questões, eu não vou deixar que isso influencie a forma como eu trato aquela criança independentemente do género que ela tem, mas eu acho que é preciso mais formação, mais informação e mais divulgação, porque acho que ainda há muitos profissionais a trabalhar com crianças, psicólogos os médicos ou o que for, que têm muito estas questões de género ainda enraizadas do Estado Novo, quase alguns será por crença própria, e por muito que as informações lhes cheguem aos olhos vão sempre fechar os olhos e virar a cara, porque se calhar não estão muito despertos. (Psicóloga social, 30-34 anos)

Os resultados do Inquérito Europeu Diversidade e Infância revelam que a falta de acesso a recursos no local de trabalho para apoiar as crianças e jovens LGBTI+ é ainda uma realidade. Para a criação de serviços mais inclusivos e sensíveis à diversidade, as/os profissionais destacam a importância do acesso a formação sobre crianças e jovens LGBTI+ e sobre população LGBTI+ no geral, a implementação de práticas e políticas LGBTI+, a construção de serviços/respostas específicas para crianças e jovens LGBTI+ e a construção de parcerias com organizações LGBTI+.

Os profissionais de saúde têm as mesmas necessidades que os docentes. Não só da tal formação, mas também de alguns materiais, normas e protocolos. Por exemplo, como falar, a questão da linguagem, que é uma coisa básica e que seria fundamental e que se devia atuar muito mais aí, e sempre do Ministério, porque tem um outro peso nas pessoas, que acabam por aderir muito mais. Portanto, acho que podia haver um trabalho próximo entre o Ministério e Associações, produzir materiais, mas que depois o Ministério lhes dava ou a Secretária de Estado [para a Igualdade], punham lá o logótipo e tal, e aquilo ficava e distribuíam eles. (Médico em pedopsiquiatria, 25-29 anos) 
O acesso a supervisão para apoiar dúvidas de casos, a divulgação de manuais para práticas profissionais inclusivas, o estabelecimento de relações com organização/coletivos LGBTI+ para prestação de apoio e assessoria são alguns exemplos identificados como práticas afirmativas em curso e que devem ser reforçadas.

\section{Impactos do adultocentrismo na regulação da diversidade sexual e de género na infância e juventude - considerações finais}

Enquanto corpo social, partilhamos a responsabilidade de combater situações de vulnerabilidade a que possam estar sujeitas crianças cujo superior interesse deve ser acautelado (Santos 2018b; 2018c). Essa responsabilidade, consagrada na Declaração dos Direitos da Criança desde $1959^{12}$, traduz-se frequentemente na reificação de um modelo cis-heteronormativo para a infância que tem como consequência prática a desproteção social e jurídica de crianças e jovens LGBTI+ ou de género diverso.

Quando olhamos para a diversidade sexual e de género na infância devemos ter presente as limitações impostas pelas normas sociais formuladas pelas pessoas adultas, que dificultam a compreensão enquanto sujeitos políticos e que influenciam a forma como incluímos as crianças nos processos democráticos ou aplicamos o quadro legal vigente (Sundhall 2017). A criança vê a sua vida determinada a partir de normas sociais formuladas pelas pessoas adultas de forma implícita e invisível, logo naturalizadas. Nesse sentido, surgem conceitos como participação da infância ou crianças-atores que pretendem destacar o reconhecimento das crianças como seres humanos completos e equivalentes aos adultos (Alanen 1988; 1992; James e Prout 1990; Qvortrup 1994; James, Jenks e Prout 1998; Hockey \& James 2003).

Outros estudos denunciam conceitos como «agência», alegando que reproduzem a norma adulta segundo a qual os direitos não são absolutos, mas devem ser alcançados (earned) (Wall 2010). Nessa linha, o conceito de childism (Pierce e Allen 1975) permite examinar de forma crítica perspetivas dominantes e desconstruir a naturalização da idade adulta.

No campo de estudos da infância, os significados e efeitos da vida adulta são pouco discutidos. A idade e as diferentes formas de regular a idade são formas comuns de criar uma ordem social (Närvänen e Näsman 2004; 2007). É por esse motivo que a idade pode ser concebida como uma ordem de poder. Figuras adultas de referência (pais, mães ou outros) poderão retirar uma vantagem injusta do poder (implícito ou explícito) que exercem sobre as crianças, e certos comporta-

12 ONU 1959. Declaração dos Direitos da Criança. Disponível em https:/ / www.dge.mec.pt/sites / default / files / ECidadania/Docs_referencia / declaracao_universal_direitos_crianca.pdf [Consultado em 2 de abril de 2021]. 
mentos adultos podem ser encorajados pela tendência dominante em ver as crianças de forma relativamente fixa e assente em relações hierárquicas, não somente com os pais ou as mães, mas com as pessoas adultas em geral.

Recordemos as reações de repúdio à Lei de Autodeterminação de Género (Lei 38/2018) a partir dos 16 anos, que dispensa intervenções cirúrgicas em pessoas intersexo menores de idade, salvaguardando situações de risco de saúde ou vida (Mariano 2020; Santos 2022). Ao respeitar a autodeterminação, Portugal colocou-se ao lado de países como a Bélgica, a Dinamarca, a Irlanda, Malta ou a Noruega, mas viu surgir movimentos de contestação que alegavam que apenas pessoas adultas poderiam tomar decisões sobre corpo, sexualidade ou género. $\mathrm{O}$ adultismo relaciona-se com esta tendência para ver as crianças e jovens como incondicionalmente subordinadas/os até atingirem a maioridade jurídica.

A diversidade sexual e de género não é exclusiva da população adulta. Daí que seja fundamental reconhecer que as experiências de crianças e jovens constituem conhecimento e que, como tal, devem informar medidas que visam garantir o superior interesse da criança. Neste ponto, importa sublinhar que o superior interesse da criança LGBTI+ tem sido sistematicamente lesado ou silenciado. Acresce que a própria noção de infância tem sido instrumentalizada ao serviço de um sistema político cis-heteronormativo (Stockton 2009; Robinson 2012; McClelland e Hunter 2013; Cardoso 2018). Preciado alerta para a construção de uma ideia de família política e uma noção de criança submetida à norma heterossexual e que se considera como tal desde o princípio, privando-a de qualquer forma de resistência e de utilizar e expressar o seu corpo de forma livre. Preciado $(2013,97)$ diz-nos que

é impossível uma criança se rebelar politicamente contra um discurso dos adultos: a criança é sempre um corpo ao qual não se reconhece o direito de governar. Quem defende então o direito das crianças que saem da norma de género e sexual? Onde está o direito à autodeterminação de género e sexualidade nas crianças e jovens?

Estando as instituições - e práticas profissionais - influenciadas por um sistema estrutural que reproduz valores cis-heteronormativos, são também elas palco de violências e fenómenos de marginalização que comprometem a igualdade no acesso à educação (Evans e Rawlings 2019; IGLYO e UNESCO 2021) e à saúde (Ceatha et al. 2020; Gato et al. 2020; Wilson e Cariola 2020). Por outras palavras, a cis-heteronormatividade regula a forma como a diversidade de género e sexual é ou não «acolhida» e garantida nos diferentes espaços institucionais, produzindo diferentes efeitos transversais, interseccionais e ao longo da vida.

Como vimos, o défice de formação e a resistência à mudança têm impactos graves para o bem-estar de crianças e jovens LGBTI+. Estes impactos são transversais, interseccionais, cumulativos e ao longo da vida. De forma a minimizar estes impactos e promover espaços seguros para todas/os, urge corresponsabilizar insti- 
tuições, envolver profissionais, apostar na formação e fomentar medidas de ação afirmativa no quotidiano, em cumprimento com o desígnio de mainstreaming LGBTI+ associado à ideia vigente de um país que se projeta internacionalmente enquanto zona de liberdade LGBTI+.

Sendo a inclusão da diversidade sexual e de género em todos os âmbitos uma forma de justiça social, constitui uma obrigação do Estado no processo de restituição de direitos (Panziera 2019). Daí a importância de olharmos para as várias áreas e espaços onde crianças e jovens participam na vida pública, fomentando perspetivas e práticas profissionais menos adultocentradas e que tenham em conta as vozes e percursos diversos de crianças e jovens.

A este respeito, importa ainda salientar que a despatologização consagrada juridicamente desde 2018 com a Lei da Autodeterminação de Género desmonta a necessidade de precaver a diversidade de género na infância, juventude e vida adulta. Consequentemente, as designadas «terapias de conversão» devem ser objeto de intervenção adequada visando a sua imediata proibição enquanto prática lesiva do bem-estar de crianças e jovens LGBTI+.

\section{Referências bibliográficas}

Alanen, Leena. 1988. «Rethinking Childhood.» Acta Sociologica 31(1): 53-67. DOI: https:/ / doi.org/10.1177/000169938803100105

Alanen, Leena. 1992. Modern childhood?: Exploring the «child question» in sociology. Jyväskä: University of Jyväskä.

Almeida, Miguel. 2010. «O contexto LGBT em Portugal.»In Estudo sobre a Discriminação em Função da Orientação Sexual e da Identidade de Género, organizado por João Oliveira e Conceição Nogueira, 45-90. Lisboa: CIG. Disponível em http:/ / bibliobase.sermais. pt:8008/BiblioNET/ upload/PDF2 / 0892_ESTUDO_ORIENTACAOSEXUAL_IDENTID.pdf [Consultado em 15 de maio de 2021].

AMPLOS, e Instituto de Apoio à Criança. 2019. Guia para Familias de crianças com papéis e comportamentos de género diverso. Lisboa: CIG. Disponível em https: / / www.cig.gov. pt/area-lgbti/documentacao/ publicacoes / [Consultado em 4 de maio de 2021].

Associação para o Planeamento da Família, CIG, e Fundação Vodafone. 2019. «E se fosse de outra cor.» Disponível em http: / / esefosseoutracor.com / [Consultado em 5 de abril de 2021].

Braun, Virginia, e Victoria Clarke. 2006. «Using thematic analysis in psychology.» Qualitative Research in Psychology 3(2): 77-101. DOI: http:/ / dx.doi.org/10.1191/1478088706 qp063oa

Bryant, Karl. 2006. «Making gender identity disorder of childhood: Historical lessons for contemporary debates.» Sexuality Research E Social Policy 3(3): 23-39. DOI: https:/ / doi.org/10.1525/srsp.2006.3.3.23

Cardoso, Daniel. 2018. «Notas sobre a Criança transviada: considerações queerfeministas sobre infâncias.» Revista Periódicus 1(9): 214-233. DOI: https: / / doi.org/10.9771/ peri. v1i9.25755

Ceatha, Nerilee, Marta Bustillo, Louise Tully, Oscar James, e Des Crowley. 2020. «What is known about the protective factors that promote LGBTI+ youth wellbeing? A scoping 
review protocol.» HRB Open Research 3(11): 177. DOI: https: / doi.org/10.12688/hrbo penres.13018.2

Chevalier, Jacques, e Daniel Buckles. 2013. Handbook for Participatory Action Research, Planning and Evaluation. Ottawa: SAS2 Dialogue. Disponível em https:/ / www.betterevaluation.org/sites / default / files / Toolkit_En_March7_2013-S.pdf [Consultado em 12 de maio de 2021].

Comissão para a Cidadania e a Igualdade de Género. 2018. «Plano de ação para o combate à discriminação em razão da orientação sexual, identidade e expressão de género, e características sexuais (2018-2021).» In Estratégia Nacional para a Igualdade - Portugal + Igual (2018-2030). Disponível em https://www.cig.gov.pt/wp-content/uploads/ 2020/12/Resol_Cons_-Ministros_61_2018.pdf [Consultado em 8 de maio de 2021].

Diversity and Childhood (DaC). 2020. National Analysis on Violence against LGBTI and Gender Non-Conforming Children: Portugal. Disponível em https://www.diversityandchildhood.eu / outputs-and-publications [Consultado em 2 de abril de 2021].

Duarte Silva, Maria João, e Maria da Luz Carvalho. 2014. «Para uma Educação Sexual Corporizada: Análise situada na educação básica em Portugal.» Revista Latino-Americana de Geografia e Gênero, 5(2): 33-41. DOI: https: / / doi.org/10.5212/Rlagg.v.5.i2.0004

Ehrensaft, Diane. 2012. «From Gender Identity Disorder to Gender Identity Creativity: True Gender Self Therapy.» Journal of Homosexuality 59(3): 337-356. DOI: https: / / doi.org/1 $0.1080 / 00918369.2012 .653303$

Evans, India, e Victoria Rawlings. 2019. «"It was Just One Less Thing that I Had to Worry about": Positive Experiences of Schooling for Gender Diverse and Transgender Students.» Journal of Homosexuality 68(9): 1489-1508. DOI: https: / / doi.org/ 10.1080 / 00 918369.2019.1698918

Fahs, Breanne. 2014. "Freedom to" and "freedom from": A new vision for sex-positive politics.» Sexualities 17(3): 267-290. DOI: https: / / doi.org/10.1177/1363460713516334

Flick, Uwe. 2005. "Triangulation in Qualitative Research.» In A Companion to Qualitative Research, organizado por Uwe Flick, Ernst von Kardorff, e Ines Steinke, 178-183. London: Sage.

Gato, Jorge, Daniela Leal, Carla Moleiro, Telmo Fernandes, Diogo Nunes, Inês Marinho, Oren Pizmony-Levy, e Cody Freeman. 2020. «"The Worst Part Was Coming Back Home and Feeling Like Crying": Experiences of Lesbian, Gay, Bisexual and Trans Students in Portuguese Schools.» Frontiers in Psychology 10(2936): 1-10. DOI: https: / / doi.org/10.3389/fpsyg.2019.02936

Green, Fiona, e May Friedman, ed. 2013. Chasing Rainbows: Exploring Gender Fluid Parenting Practices. Bradford, ON, Canada: Demeter Press.

Hines, Sally, e Ana Cristina Santos. 2018. «Trans* policy, politics and research: The UK and Portugal.» Critical Social Policy 38(1): 35-56. DOI: https://doi.org/10.1177/0261018 317732880

Hockey, Jenny, e Allison James. 2003. Social Identities Across the Life Course. Basingstoke: Palgrave Macmillan.

Human Rights Campaign (HRC). 1996. Supporting \& Caring for Transgender Children. Washington, D.C.: Human Rights Campaign. Disponível no endereço http:/ / hrc.im/ supportingtranschildren [Consultado em 3 de março de 2021].

IGLYO, e UNESCO Global Education Monitoring Report Team. 2021. «Don't look away: No place for exclusion of LGBTI students.» Policy Paper 45. Disponível no endereço https: / / en.unesco.org/gem-report/LGBTIdontlookaway [Consultado em 17 de abril de 2021]. 
ILGA Europe. 2021. Rainbow Europe Map. Disponível em https://www.ilga-europe.org/ rainboweurope/2021 [Consultado em 17 de abril de 2021].

ILGA Portugal. 2019. Relatório Anual: Discriminação contra pessoas LGBTI+. Disponível em https: / / ilga-portugal.pt / ficheiros / pdfs / observatorio/ILGA_Relatorio_Discrimina cao_2019.pdf [Consultado em 8 de junho de 2021].

James, Allison, e Alan Prout, ed. 1990. Constructing and Reconstructing Childhood: Contemporary Issues in the Sociological Study of Childhood. London: Falmer Press.

James, Allison, Chris Jenks, e Alan Prout. 1998. Theorizing Childhood. Cambridge: Polity Press.

Mariano, Gustavo. 2020. «Human Rights Discourses in the Production of Politics around Gender and Sexuality Education in Portugal.» Dissertação de doutoramento em curso, CES-UC.

Matos, Margarida G. de, Marta Reis, Lúcia Ramiro, José Pais Ribeiro, e Isabel Leal. 2014. «Educação sexual em Portugal: legislação e avaliação da implementação nas escolas.» Psicologia, Saúde EDoenças15(02):335-355.DOI:https: / / doi.org/10.15309/14psd150203

Maxwell, Joseph. 1996. Qualitative Research Design: An Interactive Approach. Los Angeles: SAGE.

McClelland, Sara, e L. E. Hunter. 2013. «Bodies That Are Always Out of Line: A Closer Look at "Age Appropriate Sexuality".» In The Moral Panics of Sexuality, organizado por Breanne Fahs, Mary Dudy e Sarah Stage, 59-76. London: Palgrave Macmillan.

Mínguez, Almudena, e Isabella Crespi. 2017. «Future perspectives on work and family dynamics in Southern Europe: The importance of culture and regional contexts.» International Review of Sociology 27(3): 389-393. DOI: https: / / doi.org/10.1080 / 0390670 1.2017.1378153

Möser, Cornelia, Jennifer Ramme, e Judit Takács, ed. 2022. Paradoxical Right-Wing Sexual Politics in Europe. Basingstoke: Palgrave Macmillan.

Närvänen, Anna-Liisa, e Elisabet Näsman. 2004. «Childhood as Generation or Life Phase?» Young 12(1): 71-91. DOI: https: / / doi.org/10.1177/1103308804039637

Närvänen, Anna-Liisa, e Elisabet Näsman. 2007. «Age order and children's agency.» In Childhood, generational order and the welfare state: Exploring children's social and economic welfare, organizado por Helmut Wintersberger, Leena Alanen, Thomas Olk, e Jens Qvortrup, 225-253. Odense: University Press of Southern Denmark.

OECD. 2019. Social Indicators: Society at a Glance 2019 - A spotlight on LGBT People. Disponível em https: / / www.oecd.org/ social/ society-at-a-glance-19991290.htm [Consultado em 14 de maio de 2021].

Ordem dos Psicólogos Portugueses. 2017. Guia Orientador da Intervenção Psicológica com Pessoas Lésbicas, Gays, Bissexuais e Trans (LGBT). Disponível em https: / / www.ordem dospsicologos.pt/ficheiros / documentos/guidelines_opp_lgbt_marco_2017.pdf [Consultado em 1 de maio de 2021].

Panziera, Andy. 2019. «Políticas públicas de acompañamiento a las infancias trans y no-binarias: Propuestas, avances y desafíos para una democracia participativa.» Revista de Educación 18: 331-338. Disponível em https://fh.mdp.edu.ar/revistas/ index.php/r_educ/article/view/3764 [Consultado em 8 de junho de 2021].

Pierce, Chester, e Gail Allen. 1975. «Childism.» Psychiatric Annals 5(7): 15-24. DOI: https:/ / doi.org/10.3928/0048-5713-19750701-04

Preciado, Paul. 2013. «Quem defende a criança queer?» Traduzido por Fernanda Nogueira. Jangada 1: 96-99. Disponível em https://www.revistajangada.ufv.br/index.php/ Jangada/article/view/17 [Consultado em 1 de abril de 2021]. 
Pyne, Jake. 2014. «Gender independent kids: A paradigm shift in approaches to gender non-conforming children.» Canadian Journal of Human Sexuality 23(1): 1-8. DOI: http: / / dx.doi.org/10.3138/cjhs.23.1.CO1

Qvortrup, Jens. 1994. «Childhood matters: An introduction.»In Childhood Matters. Social Theory, Practice and Politics, organizado por Jens Qvortrup, Marjatta Bardy, Giovanni Sgritta, e Helmut Wintersberger. Aldershot: Avebury.

rede ex aequo. 2019. Relatório do Projeto de Educação 2016-2018. Disponível em https: / / www. rea.pt/relatorioprojetoeducacao/ [Consultado em 18 de maio de 2021].

rede ex aequo. 2020. Relatório do Projeto de Educação 2019. Disponível em https: / / www.rea. pt / relatorioprojetoeducacao/ [Consultado em 3 de maio de 2021].

Robinson, Kerry H. 2012. “"Difficult citizenship”: The precarious relationships between childhood, sexuality and access to knowledge.» Sexualities 15(3-4): 257-276. DOI: https: / / doi.org/10.1177 / 1363460712436469

Roseneil, Sasha, Isabel Crowhurst, Tone Hellesund, Ana Cristina Santos, e Mariya Stoilova, ed. 2020. The Tenacity of the Couple-Norm: Intimate citizenship regimes in a changing Europe. London: UCL Press.

Saavedra, Luisa, Conceição Nogueira, e Sara Magalhães. 2010. «Discursos de jovens adolescentes portugueses sobre sexualidade e amor: implicações para a educação sexual.» Educação \& Sociedade, 31(110): 135-156. DOI: https: / / doi.org/10.1590/S0101-733020 10000100008

Saleiro, Sandra. 2017. «Diversidade de género na infância e educação: contributos para uma escola sensível ao trans(género).» ex aequo 36: 149-165. DOI: https: / / doi.org / 10.22355/ exaequo.2017.36.09

Santos, Ana Cristina. 2013. Social Movements and Intimate Citizenship in Southern Europe. Basingstoke: Palgrave.

Santos, Ana Cristina. 2018a. «Luta LGBTQ em Portugal: Duas décadas de histórias, memórias e resistências.» Revista TransVersos 14: 36-51. DOI: https://doi.org/10.12957/ transversos.2018.38654

Santos, Ana Cristina. 2018b. «Repronormativity and its Others: Queering Parental Love in Times of Culturally Compulsory Reproduction.» Analize: Journal of Gender and Feminist Studies 11: 199-215. Disponível em http://www.analize-journal.ro/library/files/ numarul_11/11_10_ana_cristina_santos_199-215.pdf [Consultado em 8 de junho de 2021].

Santos, Ana Cristina. 2018c. «Quem defende o superior interesse da criança trans?» Público, 27 de abril. Disponível em https://www.publico.pt/2018/04/27/sociedade/opiniao/ quem-defende-o-superior-interesse-da-crianca-trans-1811700 [Consultado em 8 de junho de 2021].

Santos, Ana Cristina. 2022. «Nothing from them - LGBTQI+ rights and Portuguese exceptionalism in troubled times.» In Paradoxical Right-Wing Sexual Politics in Europe, organizado por Cornelia Möser, Jennifer Ramme, e Judit Takács. Basingstoke: Palgrave Macmillan (no prelo).

Schneider, Erik. 2013. An insight into respect for the rights of trans and intersex children in Europe. Council of Europe. Disponível em https:/ / rm.coe.int/168047f2a7 [Consultado em 8 de junho de 2021].

Stockton, Kathryn B. 2009. The Queer Child, or Growing Sideways in the Twentieth Century. Durham: Duke University Press.

Suess, Amets. 2020. «Trans health care from a depathologization and human rights perspective.» Public Health Reviews 41(3). DOI: https: / / doi.org/10.1186/ s40985-020-0118-y 
Sundhall, Jeanette. 2017. «A Political Space for Children? The Age Order and Children's Right to Participation.» Social Inclusion 5(3). DOI: https:/ / doi.org/10.17645/si.v5i3.969

Torres, Anália, Rita Mendes, e Tiago Lapa. 2008. «Families in Europe.» Portuguese Journal of Social Science 7(1):49-84. DOI: https: / / doi.org/10.1386/pjss.7.1.49_1

UE. 2020. Uma União de Igualdade: Estratégia em Favor da Igualdade das Pessoas LGBTIQ 2020-2025: Construir uma União em que cada um seja livre de ser quem é. Disponível em https: / / ec.europa.eu/info/sites / default / files / lgbtiq_factsheet_2020-2025_pt.pdf [Consultado em 1 de abril de 2021].

Wall, John. 2010. Ethics in Light of Childhood. Washington, D.C.: Georgetown University Press.

Wilson, Clare, e Laura Cariola. 2020. «LGBTQI+ Youth and Mental Health: A Systematic Review of Qualitative Research.» Adolescent Research Review 5: 187-211. DOI: https:/ / doi.org/10.1007/s40894-019-00118-w

WPATH - World Professional Association for Transgender Health. 2010. «Identity recognition statement.»Disponível no endereço: https://epath.eu/wp-content/uploads/ 2018/11 / Identity-Recognition-Statement-6-6-10-on-letterhead.pdf [Consultado em 4 de abril de 2021].

Mafalda Esteves. Investigadora júnior do CES-UC desde 2015, co-coordenando em Portugal o projeto «Diversity and Childhood» (DaC). Mestre em Investigação e Intervenção Psicossocial pela Universitat de Barcelona com uma tese sobre a importância da organização e ação comunitária na promoção de empowerment psicológico. É atualmente doutoranda em Psicologia no CIS/ISCTE-IUL onde desenvolve uma tese sobre cidadania íntima e bem-estar psicossocial na bissexualidade.

Ana Cristina Santos. Socióloga e Doutorada em Estudos de Género pela Universidade de Leeds, é investigadora principal no CES-UC e líder de projetos de investigação nacionais e internacionais sobre género, sexualidade, corpo, LGBTQI+, movimentos sociais e cidadania. Co-coordenadora do programa doutoral Human Rights in Contemporary Societies desde 2013, entre os seus livros recentes incluem-se The SAGE Handbook of Global Sexualities (Sage, 2020) e The Tenacity of the Couple Norm (UCL, 2020).

Alexandra Santos. Investigadora júnior do Centro de Estudos Sociais da Universidade de Coimbra integrada no projeto «Diversity and Childhood» (DaC). É licenciada em Serviço Social pela Universidade Católica Portuguesa e mestre em Género, Sexualidade e Teoria Queer pela Universidade de Leeds (UK).

Artigo recebido a 30 de junho e aceite para publicação em 1 de outubro de 2021. 